\section{Modelling hysteresis in vanadium dioxide oscillators}

P. Maffezzoni ${ }^{\bowtie}$, L. Daniel, N. Shukla, S. Datta, A. Raychowdhury and V. Narayanan

\begin{abstract}
An original circuit-level model of two-terminal vanadium dioxide electron devices exhibiting electronic hysteresis is presented. Such devices allow realisation of very compact relaxation nano-oscillators that potentially can be used in bio-inspired neurocomputing. The proposed model is exploited to determine the parameters, values that ensure stable periodic oscillations.
\end{abstract}

Introduction: Vanadium dioxide $\left(\mathrm{VO}_{2}\right)$ is a correlated electron material that exhibits abrupt insulator-to-metal (IMT) and metal-to-insulator (MIT) phase transitions under the application of a critical electric field (transitions may also be triggered by other stimuli, such as strain and thermal excitation) [1]. These phase transitions correspond to a large and abrupt change in electrical conductivity. Recently, it has been shown that connecting a $\mathrm{VO}_{2}$ device with a resistor and a capacitor, of proper resistance and capacitance values, it is possible to fabricate very compact relaxation nano-oscillators [2]. The simple fabrication flow, the easy scalability with the possibility to achieve high packaging density, make $\mathrm{VO}_{2}$ nano-oscillators promising candidates to integrate a large array of coupled oscillators for bio-inspired neurocomputing [2,3].

However, electrical measurements reveal that the switching-like behaviour of a two-terminal $\mathrm{VO}_{2}$ comes at the expense of a hysteresis with the IMT and the MIT transition being driven by two different critical electric field values. In this Letter, we provide, for the first time, a circuit-level model of the hysteresis mechanism which is a key to predicting and controlling the dynamics of relaxation oscillators built on $\mathrm{VO}_{2}$ devices. We show how the proposed model, after being tuned with experimental data, allows us to predict the parameter values for which relaxation oscillation occurs.

Circuit-level model of $\mathrm{VO}_{2}$ device: The $I-V$ current-voltage characteristic of a two-terminal $\mathrm{VO}_{2}$ exhibits a typical hysteretic behaviour [2] (see Fig. 2). For relatively low voltages, the device has an insulator-like behaviour with $I=V / R_{\text {ins }}$, where $R_{\text {ins }}$ is the resistance in the insulator state. When the electric voltage approaches the critical value $V_{\mathrm{H}}$, a IMT occurs and the current $I$ abruptly increases. This transition is abrupt but not instantaneous with a finite intrinsic electronic switching time [4]. In the metallic state, we have that $I=V / R_{\text {met }}$ with a much smaller resistance $R_{\text {met }} \ll R_{\text {ins }}$. In this condition, to bring the device back to the insulator state, the voltage should be reduced under the lower value $V_{\mathrm{L}}<V_{\mathrm{H}}$ at which the inverse MIT occurs.

To model this hysteretic behaviour, we adopt the 'driving-point equivalent' circuit [5] with abrupt (but not instantaneous) transitions described in Fig. 1. In the model, the 'state' (i.e. insulator or metal) of the device is decided by a voltage comparator (CMP) and it is stored into the intrinsic capacitor $C_{\mathrm{o}}$. The value of the product $\tau_{\mathrm{o}}=R_{\mathrm{o}} \times C_{\mathrm{o}}$ is selected so as to reproduce the time constant of the phase transition detected in laboratory measurements. The CMP output voltage $V_{\mathrm{o}}$, whose value ranges from 0 to $1 \mathrm{~V}$, is transferred by a controlled voltage source at the CMP input terminal + , where it selects the value of $V^{+}$between the two critical levels $V_{\mathrm{L}}$ and $V_{\mathrm{H}}$. The device current is given by the current $I=I_{\mathrm{f}}=G_{\mathrm{f}} V_{\mathrm{f}}$ that flows into the feedback resistor $R_{\mathrm{f}}$ shown in Fig. 1. The electrical conductance of such a resistor is controlled by the state voltage $V_{\mathrm{c}}$ as follows

$$
G_{\mathrm{f}}=G_{\min }\left(1-V_{\mathrm{c}}\right)+G_{\max } V_{\mathrm{c}}
$$

where $G_{\min }=1 / R_{\text {ins }}$ and $G_{\max }=1 / R_{\operatorname{met}} \gg G_{\min }$ are the device conductance in the insulator and metal state, respectively. In the insulator state, the CMP output is $V_{\mathrm{o}}=1 \mathrm{~V}$ and $V_{\mathrm{c}}=0$ (at the DC regime). In this state, $V^{+}=V_{\mathrm{H}}$ and $G_{\mathrm{f}}=G_{\min }$. Hence, if $V$ is gradually increased till to approach $V_{\mathrm{H}}$, it initiates a IMT. After the device has completed its transition to the metallic state, the CMP output becomes $V_{\mathrm{o}}=0 \mathrm{~V}$ and $V_{\mathrm{c}}=1$. In this state, $G_{\mathrm{f}}=G_{\mathrm{Max}}$ and $V^{+}=V_{\mathrm{L}}$. Thus, it is only when $V$ is reduced below $V^{+}=V_{\mathrm{L}}$ that a reverse MIT can occur completing the cycle of the hysteresis characteristic. Fig. 2 shows the $I-V$ characteristics obtained by simulating the circuit-level model in Fig. 1 for a $2 \mu \mathrm{m}$-length $\mathrm{VO}_{2}$ device. The parameters of the model were extracted by fitting the experimental curve of the device as follows: $R_{\text {ins }}=$ $50 \mathrm{k} \Omega, R_{\text {met }}=1 \mathrm{k} \Omega, V_{\mathrm{L}}=0.5 \mathrm{~V}, V_{\mathrm{H}}=5.9 \mathrm{~V}$ and $\tau_{\mathrm{o}}=100 \mathrm{~ns}$. The device is connected in series to a DC voltage source, sweeping from 0 to $7 \mathrm{~V}$, and to a resistance $R_{\mathrm{s}}$. Fig. 2 shows the $I-V$ curves for the two cases: (i) zeros series resistance $R_{\mathrm{s}}=0$ and (ii) finite series resistance $R_{\mathrm{S}}=200 \Omega$. Simulations correctly predict the negative-slope shape of the $I-V$ curve in the presence of a finite resistance $R_{\mathrm{s}}$ [2]. We conclude that, after being tuned with the experimental data of a given $\mathrm{VO}_{2}$ device, the proposed circuit-level model is able to realistically reproduce its electronic behaviour seen at the external terminals.

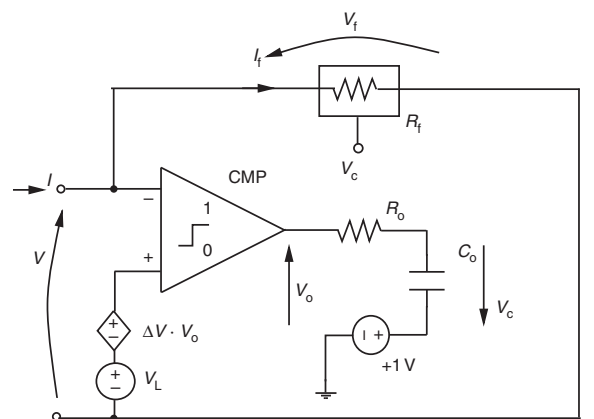

Fig. 1 Device model

$\Delta V=V_{\mathrm{H}}-V_{\mathrm{L}}$ gives width of voltage hysteresis

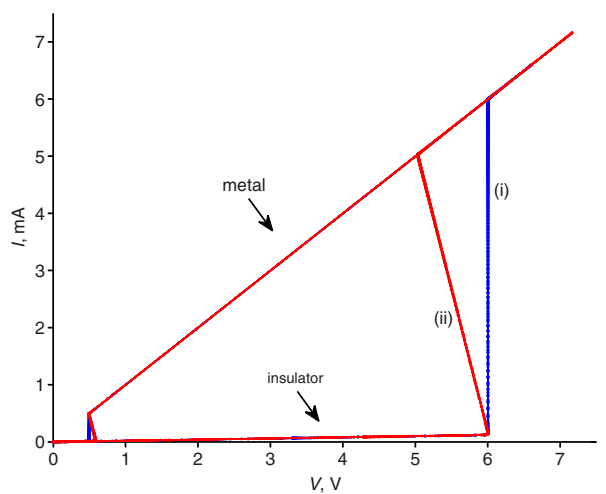

Fig. 2 Simulated $\mathrm{VO}_{2}$ characteristics for cases: (i) $R_{s}=0 \Omega$ and (ii) $R_{s}=$ $200 \Omega$

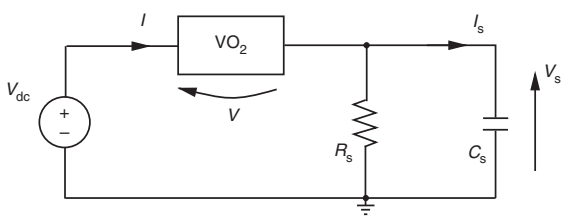

Fig. 3 Circuit of relaxation oscillator

Relaxation oscillation: The circuit of the relaxation oscillator can be obtained by connecting the $\mathrm{VO}_{2}$ device to an external resistor $R_{\mathrm{S}}$ and capacitor $C_{\mathrm{s}}$, as shown in Fig. 3. The circuit is supplied by a DC voltage source $V_{\mathrm{dc}}$. We performed extensive electrical simulations of the circuit in Fig. 3, with the $\mathrm{VO}_{2}$ device model in Fig. 1, to investigate the effect of electrical parameters. Simulations showed that a minimum supply voltage $V_{\mathrm{dc}}$ value is needed to obtain oscillations. Furthermore, they revealed that, for a given voltage supply, the occurrence of relaxation oscillations critically depends on the value of the external series resistance $R_{\mathrm{s}}$; oscillations only arise when $R_{\mathrm{s}}$ is chosen within a very precise interval of values. With the $\mathrm{VO}_{2}$ device in our example and with $V_{\mathrm{dc}}=14 \mathrm{~V}$, stable oscillations occur for $30 \mathrm{k} \Omega<R_{\mathrm{s}}<55 \mathrm{k} \Omega$. Finally, the value of the external capacitance $C_{\mathrm{s}}$ affects the frequency of the oscillation. This behaviour was fully confirmed by laboratory measurements. Fig. 4 reports the simulated and the measured $V_{\mathrm{s}}(t)$ waveforms for a relaxation oscillator with electrical parameters $V_{\mathrm{dc}}=$ $14 \mathrm{~V}, C_{\mathrm{s}}=300 \mathrm{pF}$ and $R_{\mathrm{s}}=47 \mathrm{k} \Omega$ (i.e. within the numerically-predicted oscillation interval); simulations and measurements match with excellent accuracy and yield a relaxation oscillation at $\approx 68 \mathrm{kHz}$. 


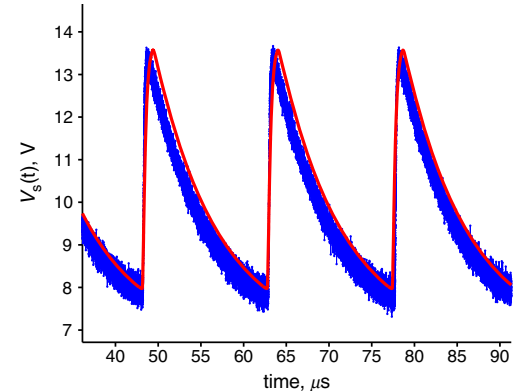

Fig. 4 Simulated (solid line) and measured (dotted line) $V_{s}(t)$ waveforms for $R_{s}=47 \mathrm{k} \Omega$

Conclusion: In this Letter, the electrically induced hysteresis mechanism in $\mathrm{VO}_{2}$ devices has been modelled for the first time with a drivingpoint equivalent circuit. The circuit can incorporate the experimentally characterised critical voltages, variable device resistances and switching time of the hysteretic response. We have shown how the proposed model enables realistic electrical simulations of oscillators built on the hysteretic device and provides guidance on how to achieve a robust design. It is believed that the circuit-level model will play a fundamental role in designing large arrays of $\mathrm{VO}_{2}$ oscillators.

(C) The Institution of Engineering and Technology 2015

19 January 2015

doi: $10.1049 / \mathrm{el} .2015 .0025$
One or more of the Figures in this Letter are available in colour online.

P. Maffezzoni (Politecnico di Milano, Milan, Italy)

凶 E-mail: pmaffezz@elet.polimi.it

L. Daniel (Massachusetts Institute of Technology, Cambridge, MA, USA)

N. Shukla, S. Datta and V. Narayanan (Pennsylvania State University, University Park, PA, USA)

A. Raychowdhury (Georgia Institute of Technology, Atlanta, GA, USA)

\section{References}

1 Sugimoto, N., Onoda, S., and Nagaosa, N.: 'Field-induced metal-insulator transition and switching phenomenon in correlated insulators', Phys. Rev. B, 2008, 78, (15), p. 155104

2 Datta, S., Shukla, N., Cotter, M., Parihar, A., and Raychowdhury, A.: 'Neuro inspired computing with coupled relaxation oscillators'. Design Automation Conf. San Francisco, CA, USA, June 2014, pp. 1-6

3 Shukla, N., Parihar, A., Freeman, E., et al.: 'Synchronized charge oscillations in correlated electron systems'. Nature, Sci. Rep, 2014, 4, doi 10.1038/srep04964

4 Kar, A., Shukla, N., Freeman, E., et al.: 'Intrinsic electronic switching time in ultrathin epitaxial vanadium dioxide thin film', Appl. Phys. Lett., 2013, 102, (7), p. 72106

5 Hu, C.J.: 'Self-sustained oscillation in an RH-C or RH-L circuit containing a hysteresis resistor RH', IEEE Trans. Circuits Syst., 1986, 33, (6), pp. 636-641 
Copyright of Electronics Letters is the property of Institution of Engineering \& Technology and its content may not be copied or emailed to multiple sites or posted to a listserv without the copyright holder's express written permission. However, users may print, download, or email articles for individual use. 